\title{
Modification of Generalization of Equivalence Relation
}

\author{
Sarat K. Parhi \\ Vijayanjali Institute of Technology, Khantapada,Balasore,Odisha-756043
}

\begin{abstract}
In this paper contains description of certain relations called partial equivalence relations. Moreover ,it includes the formal algorithms for determining the order of relations like partial reflexive, partial symmetric and partial transitive relations. Some examples are also presented for the clarification of these concept.
\end{abstract}

Key Words:-Partial reflexive, Partial symmetric and Partial transitive .

\section{Introduction:}

Mathematical meaning of the word "Relation" can be interpreted as the association or dissociation ,interaction or interconnectedness of elements of two sets. The word "Relation" may be interpreted as the quantification of propositions .In mathematics, various form of relations available. Most commonly used relations are "Preorder","Order relation", "Partial order relation", "Strict ordering relation". "Binary relation", "n-arrey Relation", "Equivalence relation",Fuzzy set and Rough set relation etc. Whatever may be the forms ,any relation is a special type of set that connects some other sets in a proper way.

In this paper I want to discuss certain type of modification of generalization of equivalence relations. The Fuzzy relation describes some of the incomplete equivalence relations ,but this description is insufficient .There exists certain relations, which often used in the day-to-day linguistic expressions and need more attention for the application point of view.

\section{Comparison Of Partial Equivalence Relation With Fuzzy Relations :-}

The readers who are not familiar with the partial relation may think why one should define partial relation although the definition of fuzzy relation already exists ? To answer this query, in fuzzy relation the membership function itself is a relationship between the elements of the set on which the fuzzy relation $\bar{R}$ is defined that's why the relation $\bar{R}$ is associated with another relation. Fuzzy relation and partial equivalence relation are different concepts .Those are summarized below.

\subsection{Fuzzy Relation}

a. The knowledge of the memberships of Individual elements within the domain of fuzzy relation is essential.

b. Only one membership relation is required to represent a fuzzy relation.

c. Membership function differs as per the variation of the rùles.

2.2Partial Equivalence relation a .Partial equivalence relation does not require it essentially.

$\mathrm{b}$. Among them two relations are the subset of the Cartesian product of a set is required to define a partial relation.

1

c. Partial relation neither contain a membership function nor it changes as the variation of the rules.

Example:-3.1

\section{Implementation:-}

Let us consider the grid as shown in the figure. The English alphabet B and P can traced by using this grid .The trace of $\mathrm{B}$ and $\mathrm{P}$ are

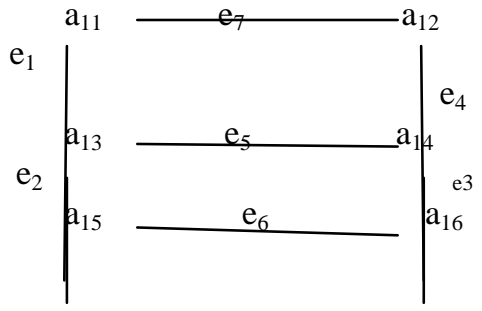

*(Fig.3.1)

Trace of $B=\left\{\left(a_{15}, a_{13}\right),\left(a_{13}, a_{11}\right),\left(a_{11}, a_{12}\right),\left(a_{12}, a_{14}\right),\left(a_{14}, a_{13}\right),\left(a_{14}, a_{16}\right),\left(a_{15}, a_{16}\right)\right\}$

Trace of $\mathrm{P}=\left\{\left(\mathrm{a}_{15}, \mathrm{a}_{13}\right),\left(\mathrm{a}_{13}, \mathrm{a}_{11}\right),\left(\mathrm{a}_{11}, \mathrm{a}_{12}\right),\left(\mathrm{a}_{12}, \mathrm{a}_{14}\right),\left(\mathrm{a}_{14}, \mathrm{a}_{13}\right)\right\}$ 
Let the relationship between $\mathrm{B}$ and $\mathrm{P}$ which can be modeled as the traverse of maximal similar path in the grid. Let us consider the trace of $B$ and $P$ are the relationships between $R_{1}$ and $R_{2}$ is $R_{12}$ respectively then $R_{12}=\left\{\left(a_{15}, a_{13}\right),\left(a_{13}, a_{11}\right),\left(a_{11}, a_{12}\right),\left(a_{12}, a_{14}\right),\left(a_{14}, a_{13}\right)\right\}$

The order of similar appearance of $\mathrm{B}$ with $\mathrm{P}$ is $\mathrm{I}$ Trace of $\mathrm{B} \cap$ Trace of $\mathrm{PI} / \mathrm{I}$ Trace of $\mathrm{PI}=5 / 5=1$

The order of partial appearance of $\mathrm{B}$ with $\mathrm{P}$ is $\mathrm{I}$ Trace of $\mathrm{B} \cap$ Trace of PI/ I Trace of BI $=5 / 7=0.7142857$.

The order of partial reflexive relation is $\min \{1,5 / 7\}=5 / 7=0.7142857$

\section{Remark:-1}

The order of partial reflexivity is greater than or equal to zero or lesser than or equal to one $i=\{1,2,3,4,5\}$ the index used for the edges $i$ on the grid for tracing $P$. $\mathrm{j}=\{1,2,3,4,5,6,7\}$ the index used for the edges $\mathrm{j}$ on the grid for tracing of $\mathrm{B}$.

Formal Algorithm:-1 The algorithm for forming matrix as follows

$=1$ if both the edges $\mathrm{e}_{\mathrm{ij}}$ are used for $\mathrm{i}=\mathrm{j}$

$$
=0 \text { if at least } e_{i} \text { and } e_{j} \text { are not used if } i \neq j
$$

\begin{tabular}{l|ll} 
& \multicolumn{2}{|c}{ Trace of $\mathrm{B} \rightarrow$} \\
& $\mathrm{e}_{1}$ & $\mathrm{e}_{2}$ \\
$\mathrm{e}_{1}$ & 1 & 0 \\
$\mathrm{e}_{2}$ & 0 & 1 \\
$\mathrm{e}_{3}$ & 0 & 0 \\
$\mathrm{e}_{4}$ & 0 & 0 \\
$\mathrm{e}_{5}$ & 0 & 0
\end{tabular}

$\begin{array}{lll}\mathrm{e}_{3} & \mathrm{e}_{4} & \mathrm{e}_{5} \\ 0 & 0 & 0 \\ 0 & 0 & 0 \\ 1 & 0 & 0 \\ 0 & 1 & 0 \\ 0 & 0 & 1\end{array}$

\section{Proposition:-3.1.1}

Every partial reflexive relation is reflexive but converse is not true.

Proof is not needed and left it.

\section{Example :-3.2}

Let us consider the grid as shown in the figure 3.1

The English alphabets $\mathrm{B}$ and $\mathrm{H}$ can be traced by using this grid .

The trace of $\mathrm{B}$ and trace of $\mathrm{H}$ are

Trace of $B=\left\{\left(a_{15}, a_{13}\right),\left(a_{13}, a_{11}\right),\left(a_{11}, a_{12}\right),\left(a_{12}, a_{14}\right),\left(a_{14}, a_{13}\right),\left(a_{14}, a_{16}\right),\left(a_{15}, a_{16}\right)\right\}$

Trace of $\mathrm{H}=\left\{\left(\mathrm{a}_{11}, \mathrm{a}_{13}\right),\left(\mathrm{a}_{13}, \mathrm{a}_{15}\right),\left(\mathrm{a}_{12}, \mathrm{a}_{14}\right),\left(\mathrm{a}_{16}, \mathrm{a}_{14}\right),\left(\mathrm{a}_{13}, \mathrm{a}_{14}\right)\right\}$

Let the relationship between $B$ and $H$ be $\mathrm{R}_{12}$, i.e ,the similarity in the appearance of $\mathrm{B}$ with $\mathrm{H}$. The relationship can be measured by enlisting all the common edges those appears in the trace of $\mathrm{B}$ and $\mathrm{H}$ in the grid..If we consider the traces of $\mathrm{B}$ and $\mathrm{H}$ in the grid. if we consider the traces of $\mathrm{B}$ and $\mathrm{H}$ as the relationships $\mathrm{R}_{1}$ and $\mathrm{R}_{2}$ is $R_{12}$ then

$\mathrm{R}_{12}=\left\{\left(\mathrm{a}_{11}, \mathrm{a}_{13}\right),\left(\mathrm{a}_{13}, \mathrm{a}_{15}\right),\left(\mathrm{a}_{12}, \mathrm{a}_{14}\right),\left(\mathrm{a}_{16}, \mathrm{a}_{14}\right),\left(\mathrm{a}_{13}, \mathrm{a}_{14}\right)\right\}$

The order of similar appearance of $\mathrm{B}$ with $\mathrm{H}=\mathrm{I}$ Trace of $\mathrm{B} \cap$ Trace of HI/ I Trace of $\mathrm{HI}=5 / 5=1$

The of partial appearance of $\mathrm{H}$ with B is I Trace of $\mathrm{B} \cap$ Trace of HI/ I Trace of BI $=5 / 7=0.7142857$

Order of partial symmetric relation is $\min \{1,5 / 7\}=5 / 7=0.7142857$

\section{Remark-2}

The order of partial symmetric is always greater then equal to zero and lesser then equal to one.

The partial symmetric relationship can be expressed in the matrix form .Here we express such algorithm by illustrating the following partial symmetric relationship of the trace of $\mathrm{H}$ with the trace $\mathrm{B}$.The edges consisting the trace of $\mathrm{H}$ are taken in the row and the edges consists of trace of $\mathrm{B}$ are taken in columns. The algorithm for forming matrix as follows.

Formal Algorithm-2

$\mathrm{e}_{\mathrm{ij}}=\mathrm{e}_{\mathrm{ji}}=1$, if both $\mathrm{e}_{\mathrm{i}}$ and $\mathrm{e}_{\mathrm{j}} \in$ Trace of $\mathrm{H} \cap$ Trace of $\mathrm{B}$ and $\mathrm{e}_{\mathrm{i}}, \mathrm{e}_{\mathrm{j}}$ are connected to at least a node . $=0$,if either any of $e_{i}$ or $e_{j}$ not $C$ Trace of $H \cap$ Trace of $B$. 


\begin{tabular}{|c|c|c|c|c|c|c|c|c|}
\hline & Trr & $\mathrm{B}$ & & & & & & \\
\hline & & $\mathrm{e}_{1}$ & $\mathrm{e}_{2}$ & $\mathrm{e}_{3}$ & $\mathrm{e}_{4}$ & $\mathrm{e}_{5}$ & $\mathrm{e}_{6}$ & $\mathrm{e}_{7}$ \\
\hline & $e_{1}$ & 1 & 1 & 0 & 0 & 1 & 0 & 0 \\
\hline Trace of $\mathrm{H} \rightarrow$ & $e_{2}$ & 1 & 1 & 0 & 0 & 1 & 0 & 0 \\
\hline $\mathrm{e}_{3}$ & 0 & 0 & 1 & 1 & 1 & 0 & 0 & \\
\hline & $\mathrm{e}_{4}$ & 0 & 0 & 1 & 1 & 1 & 0 & 0 \\
\hline & $e_{5}$ & 1 & 1 & 1 & 1 & 1 & 0 & 0 \\
\hline
\end{tabular}

Every partial symmetric relation is symmetric relation but converse is not true.

Proof:- In this matrix fig 3.3

Let $x \in$ Trace of $H$ and $y \in$ Trace of $B$.

$x R y$ is called a trace of $H R$ trace of $B$.

$\mathrm{x} \mathrm{R}$ y C (Trace of $\mathrm{H}) \mathrm{R}$ (Trace of B)

Similarly transpose of fig 3.3 is written as follows y $\mathrm{R}=>$ (Trace of $\mathrm{B}) \mathrm{R}($ Trace of $\mathrm{H}$ ) is called the transpose of the matrix.This is the complete proof .

Theorem:-3.1

The matrix associated with the partial symmetric relation $\mathrm{R}_{12}$ is equal to the similar matrix associated with the partial symmetric relation $R_{21}$ Provided $I_{1} I=I_{2} I$.

Proof:--In order to prove this theorem two cases arises

Case:-1 Suppose $I R_{1} I=I_{2} I$

Then the proof is obvious as for Formal Algorithm-2

Case:-2 I R $\mathrm{I} \neq \mathrm{IR}_{2} \mathrm{I}$

Let us consider the Sub matrix of order $\min \left\{\mathrm{I} \mathrm{R}_{1} \mathrm{I}, \mathrm{IR}_{2} \mathrm{I}\right\} \mathrm{X} \min \left\{\mathrm{I} \mathrm{R}_{1} \mathrm{I}, \mathrm{IR}_{2} \mathrm{I}\right\}$ and this sub matrix must be associated with the elements those are included from the first row and first column to min $\left\{\mathrm{I}_{1} \mathrm{I}, \mathrm{IR}_{2} \mathrm{I}\right\}$ row and column respectively. The elements those are below of the main diagonal of this sub matrix are equal. That neighborhood $\mathrm{a}_{\mathrm{ij}}=\mathrm{a}_{\mathrm{ji}}$ for $\left\{1,2,3, \ldots \min \left\{\mathrm{I} \mathrm{R}_{1} \mathrm{I}, \mathrm{IR}_{2} \mathrm{I}\right\}\right.$.

\section{Example -3.3}

Let us consider the grid as shown in the figure-3.1.The English alphabets B,H and I can be traced by using the grid.

The trace of $\mathrm{B}, \mathrm{H}$ and $\mathrm{I}$ with the relations $\mathrm{R}_{1}, \mathrm{R}_{2}$ and $\mathrm{R}_{3}$ are

Trace of $\mathrm{B}=\left\{\left(\mathrm{a}_{15}, \mathrm{a}_{13}\right),\left(\mathrm{a}_{13}, \mathrm{a}_{11}\right),\left(\mathrm{a}_{11}, \mathrm{a}_{12}\right),\left(\mathrm{a}_{12}, \mathrm{a}_{14}\right),\left(\mathrm{a}_{14}, \mathrm{a}_{13}\right),\left(\mathrm{a}_{14}, \mathrm{a}_{16}\right),\left(\mathrm{a}_{15}, \mathrm{a}_{16}\right)\right\}$

Trace of $\mathrm{H}=\left\{\left(\mathrm{a}_{11}, \mathrm{a}_{13}\right),\left(\mathrm{a}_{13}, \mathrm{a}_{15}\right),\left(\mathrm{a}_{12}, \mathrm{a}_{14}\right),\left(\mathrm{a}_{16}, \mathrm{a}_{14}\right),\left(\mathrm{a}_{13}, \mathrm{a}_{14}\right)\right\}$

Trace of $I=\left\{\left(a_{11}, a_{31}\right),\left(a_{15}, a_{13}\right)\right\}$

Let the trace of $B$ and trace of $H$ is $R_{12}$ and the trace between $H$ and $I$ is $R_{23}$. Then the trace of $B$ and trace of $I$ is $\mathrm{R}_{13}$.

The order of similar appearance of $\mathrm{B}$ with $\mathrm{H}$ is I Trace of $\mathrm{B} \cap$ Trace of $\mathrm{HI} / \mathrm{I}$ Trace of $\mathrm{H} \mathrm{I}=5 / 5=1$

The order of similar appearance of B with I is I Trace of B $\cap$ Trace of I I / I Trace of I I $=2 / 2=1$

The order of partial appearance of I with B is I Trace of I $\cap$ Trace of B I / I Trace of B I $=2 / 7=0.2857143$

The order of partial transitive relation is $\min \{1,1,2 / 7\}=2 / 7=0.2857143$

Formal Algorithm:-3 $e_{i j}=1$, if both the edges $e_{i}$ and $e_{j}$ has a link for $i=j$

$=0$,if both the edges of $e_{i}$ and $e_{j}$ has no link.

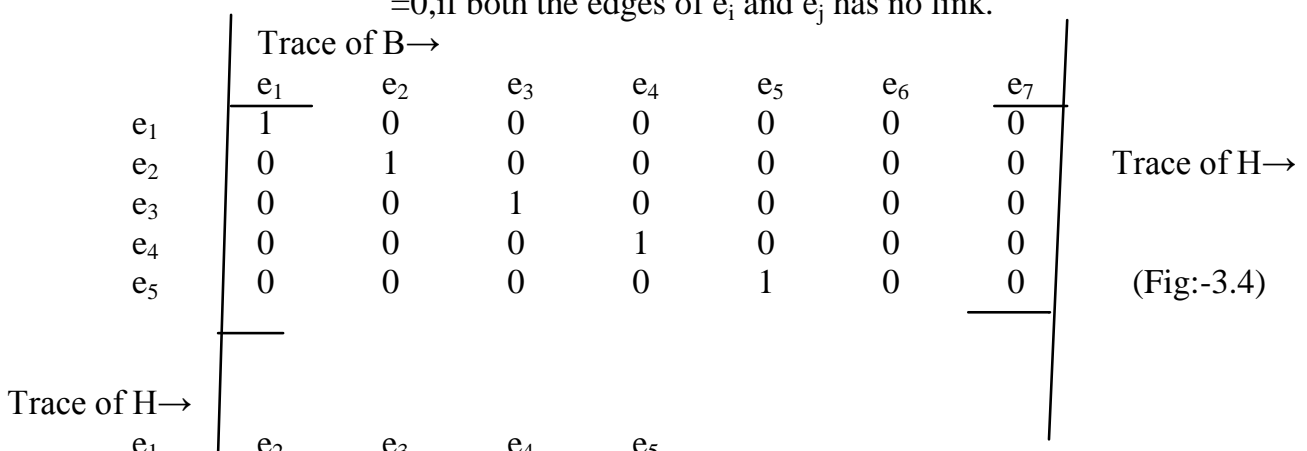

(Fig:-3.5) 


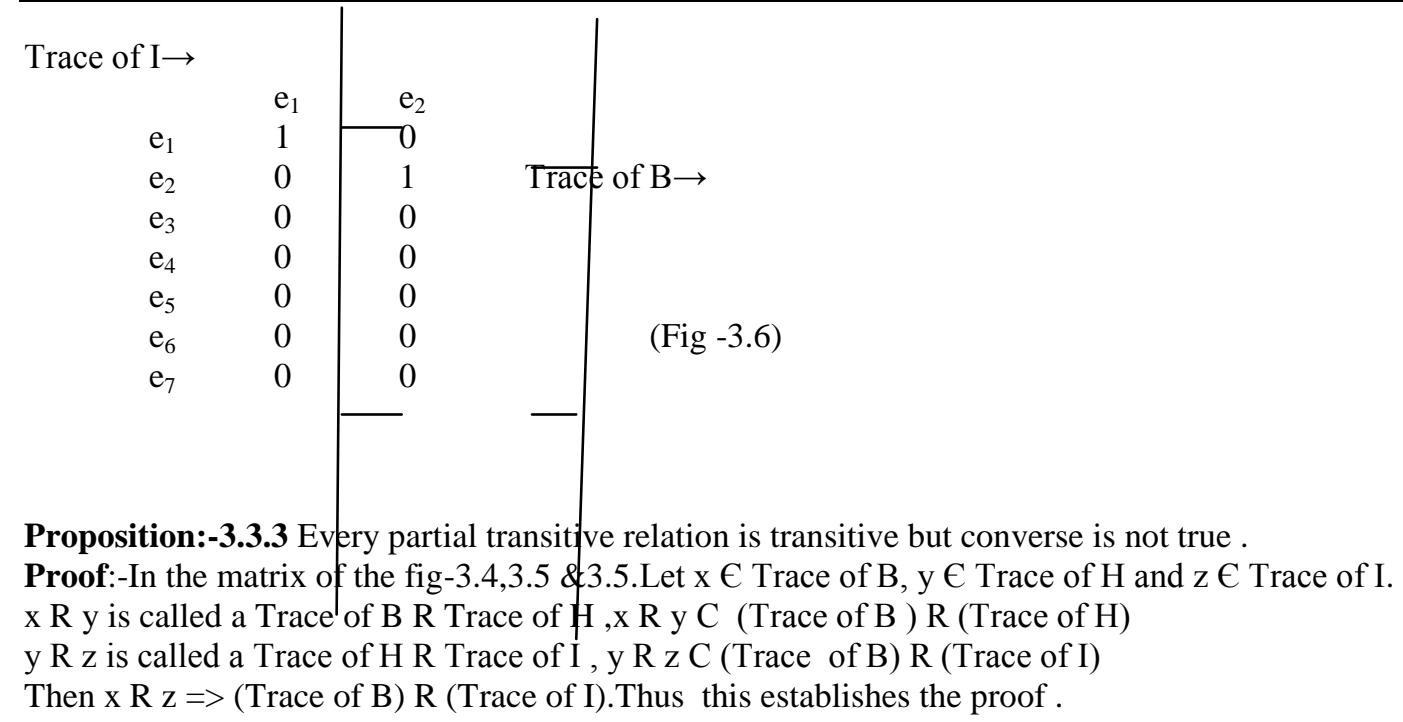

\section{Conclusion :-}

This paper defines theorem and propositions to help the many development of formulae. Using formal algorithm to studied many theoretical and implicational problem in near future. The order of partial reflexive ,partial symmetric and partial transitive to give better clarification of the concept.

\section{References:}

[1]. J. Klir,George, \& A. Floger,Tina ,Fuzzy Sets, Uncertainty and Information ,Prentice-Hall of India Private Limited,2008,P.65-105

[2]. Parhi,S.K ,Das,L, "Generalization of Equivalence Partial Relation" Odisha Mathematical Society Annual Conference abstract of National Conference on Recent Trends in Mathematics,27-28, may -2001,P.3

[3]. Parhi,S.K. , Das,L., " Generalization of Equivalence Relation " Submitted to International journal of Fuzzy Information Engineering, China 